Ann. Biol. anim. Bioch. Biophys., 1978, 18 (4), 825-829.

\title{
Etude du contrôle hypothalamique de la fonction gonadotrope chez Gambusia sp. (poisson téléostéen) par la technique des greffes
}

\author{
par P. CHAMBOLLE, O. KAH, Madeleine OLIVEREAU * \\ Laboratoire de Biologie Animale A, Centre de Morphologie expérimentale du C. N. R. S. \\ Université de Bordeaux I, avenue des Facultés, 33405 Talence \\ * Institut Océanographique, 75005 Paris.
}

Summary. Gonadotropic function in the teleost Gambusia sp. A study of hypothalamic control using grafts.

In the viviparous poeciliid, Gambusia sp., long-term (3 to 12 months) pituitary autografts permitted mainfenance of the ovarian structure and normal functioning of vitellogenesis. It is likely that hypothalamic stimulation of gonadotropic cells of the graft was through a neuro-vascular tract.

\section{Introduction.}

Afin de préciser les modalités du contrôle hypothalamique de la fonction gonadotrope chez Gambusia, nous avons étudié l'activité de l'hypophyse autotransplantée, déconnectée de l'hypothalamus, mais, néanmoins, soumise à l'influence éventuelle des récepteurs. L'autogreffe d'hypophyse chez le Cyprin (Johansen, 1967) ou l'homogreffe chez Poecilia (Ball et al. 1965) entraîne en 1 ou 2 mois une régression des gonades et des cellules gonadotropes du greffon. Chez Gambusia (Chambolle, 1977), des autogreffes pratiquées en début de gestation ne permettent pas à la vitellogenèse de redémarrer après la parturition (1 mois après la greffe). Nous avons repris l'étude de l'influence d'une autotransplantation hypophysaire à long terme sur l'activité ovarienne.

\section{Matériel et méthode.}

Nous avons utilisé le Poeciliidae vivipare Gambusia sp. Sa période de reproduction s'étend de mai à septembre dans les conditions naturelles de notre région et 4 à 5 cycles de reproduction se succèdent sans interruption. Au moment de la parturition, l'ovaire ne contient aucun ovocyte en cours de vitellogenèse. Celle-ci débute immédiatement après. La période de repos sexuel s'étend d'octobre à avril, les ovaires ne contiennent alors que des ovocytes I ef II. 
Nous avons hypophysectomisé 50 femelles par voie buccale. Ces poissons consi'dérés comme témoins des « greffés » sont sacrifiés chaque mois pendant un an pour Tefude histologique de l'ovaire. Témoins ef opérés sont élevés à $25{ }^{\circ} \mathrm{C}$ avec une photopériode naturelle dans un mélange de $2 / 3$ d'eau douce et de $1 / 3$ d'eau de mer, permettant une survie parfaite (Chambolle, 1966).

Chez 90 animaux, nous avons pratiqué une autotransplantation de l'hypophyse, soit en la greffant dans la musculature caudale (chez 30 animaux) (fig. 1), soit en la réintroduisant dans la selle turcique (chez 60 animaux) (fig. 2). Chez ces animaux sacrifiés comme les témoins, nous avons fait l'étude histologique de l'ovaire ef du greffon hypophysaire (coloration avec le Cleveland Wolfe). Nous avons toujours vérifié la réussite de l'hypophysectomie par un contrôle histologique. Les cas d'ablation partielle n'ont pas dépassé 2 p. 100 des animaux opérés et ont été éliminés.

\section{Résultats.}

Hypophysectomie : l'ablation pratiquée pendant la période de repos sexuel entraîne une réduction progressive du stroma ovarien qui, suivant les animaux, semble être maximale après 6 à 12 mois (fig. 3 et 4). Les ovocytes I et II ne dégénèrent pas. En bordure de la cavité ovarienne, l'épithélium est très réduit.

L'ablation pratiquée pendant la période d'activité sexuelle provoque, en outre, la dégénérescence de tous les ovocytes en vitellogenèse. Ces résultats sont en accord avec de nombreux travaux antérieurs montrant que l'hypophyse est indispensable au déclenchement et au maintien de la vitellogenèse. L'hypophyse est aussi indispensable pour conserver une structure normale du stroma ef de l'épithélium ovarien chez Gambusia.

Greffe d'hypophyse : chez des animaux opérés au cours de la période de repos sexuel, la vitellogenèse se déclenche en même temps que celle des animaux normaux non opérés, lorsque le greffon a été placé dans la selle turcique et à condition, toutefois, que la greffe ait été pratiquée au minimum deux mois avant le début de la période de reproduction (résultats sur 25 animaux). Lorsque le greffon est placé dans la musculature caudale, le même phénomène s'observe (résultats sur 10 animaux) mais avec un retard plus ou moins prononcé suivant les animaux ( 1 mois en moyenne). Quel que soit le type de greffe pratiqué, la vitellogenèse se déroule normalement (fig. 5). Chez certains animaux conservés suffisamment longtemps (plus de 3 mois), nous avons pu obtenir une gestation et une parturition normales. L'histologie des

FIG. 1. - Greffon d'hypophyse dans la musculature caudale, 12 mois après la greffe $(G=140)$.

FIG. 2. - Greffon d'hypophyse dans la selle furcique, 5 mois après la greffe $(G=168)$.

FIG. 3. - Ovaire 10 mois oprès hypophysectomie (comparez avec la fig. 4) $(G=108)$.

FIG. 4. - Ovaire d'un animal témoin pendant la période de repos sexuel $(G=62)$.

FIG. 5 et 6. - Ovaire après autogreffe d'hypophyse dans lo musculature caudale 12 mois après la greffe $(G=148)$ et cellules gonadotropes du greffon du même animal ( $G=1530)$. 
greffons nous a permis d'identifier foutes les catégories cellulaires et, en particulier, les cellules gonadotropes qui paraissent très actives avec un noyau arrondi à nucléole bien visible ef un cytoplasme bien granulé (fig. 6).

Chez des animaux opérés durant la période de reproduction, les résultats sont plus difficiles à interpréter. Dans un premier temps, l'ablation a pour conséquence l'atrésie des ovocytes en vitellogenèse. Dans un deuxième temps, on peuł observer une reprise de la vitellogenèse qui se fait au début de la période de reproduction suivante, soit 7 à 8 mois après l'opération. II est fréquent d'observer dans l'ovaire la présence d'ovocytes dont l'atrésie n'est pas terminée à côté d'ovocyłes en vitellogenèse.

Les résultats les plus démonstratifs et les plus facilement interprétables nous paraissent être ceux obtenus après ablation au cours de la période de repos sexuel. Dans ce cas lors de l'opération, les cellules gonadotropes ef l'ovaire sont au repos et il apparaît clairement que, au moment de la reprise normale de l'activité sexuelle chez cette espèce, les cellules gonadotropes, quelle que soit la lacalisation du greffon, sont capables de stimuler l'activité ovarienne, en particulier la vitellogenèse.

\section{Discussion.}

Les résultats obtenus chez Gambusia diffèrent de ceux signalés chez Poecilia (Ball et al., 1965). Les différences observées semblent dues à la longue durée de nos expériences et aux nombreux essais pratiqués à des époques différentes de l'année. Jusqu'à présent, nous n'avons pu obtenir la reprise de la vitellogenèse après autogreffe d'hypophyse dans la musculature caudale, qu'au moment de la reprise de l'activité sexuelle de l'espèce, au début du printemps. II semble qu'à cette époque, les cellules gonadotropes sont plus sensibles à l'action d'un facteur hypothalamique dont le taux serait alors maximal. Cette hypothèse est en accord avec les travaux de Weil ef al. (1975), chez la Carpe.

Nos résultats sont à rapprocher de ceux obtenus chez le Rat. Dans le cas de greffes multiples d'hypophyse, les greffons peuvent sécréter de petites quantités de LH et FSH (Lu et al., 1977). La présence d'une LH-RH, bien démontrée chez les Vertébrés supérieurs, semble vérifiée chez la Carpe (Breton et Weil, 1973). Mais son mode d'action est peu connu. Des cellules à LH-RH ont été mises en évidence dans le télencéphale de la Truite, mais le trajet exact des axones n'a pu être précisé (Goos et Murathanoglu, 1977). Le transport de cette hormone pourrait se faire par voie neuroglandulaire (Zambrano, 1972 ; Ekengren, 1975). Deux types de fibres nerveuses (A ef B) établissent des contacts synaptiques avec les cellules gonadotropes de Poecilia (Peute ef al., 1976). Les fibres B proviendraient du noyau latéral de tuber qui paraît être impliqué dans le contrôle de la reproduction (Peter, 1970 ; Zambrano, 1972). Cette zone est en outre très sensible à la rétroaction des œstrogènes sur l'activité gonadotrope chez le Cyprin (Billard ef Peter, 1977). L'existence d'une voie neurovasculaire a cependant été envisagée par Sathyanesan (1970, 1971), Fridberg et Ekengren (1977).

S'il existe une stimulation hypothalamique des cellules gonadotropes par voie nerveuse chez Gambusia, dans nos expériences de greffe, le transport de la LH-RH par voie strictement nerveuse n'est plus envisageable. Il faut donc admettre la possibilité d'un transport par voie neuro-vasculaire, le chaînon vasculaire devenant alors le plus important. 
Une autre hypothèse pour expliquer nos résultats ne peut être écartée : celle d'une sécrétion autonome de gonadotrophine s'établissant après greffe de longue durée similaire à la sécrétion de FSH observée lors de culture d'hypophyse de Rat pendant 18 semaines à 3 ans (Sheridan et Pasteels, 1977).

En conclusion, chez Gambusia des autogreffes d'hypophyse permettent le maintien de la structure ovarienne et le déroulement normal de la vitellogenèse. Il est probable que la stimulation hypothalamique des cellules gonadotropes du greffon se fait par voie neuro-vasculaire.

Symposium sur la Reproduction des Poissons Paimpont, France, 19-21 septembre 1977.

\section{Références}

BALL J. N., OLIVEREAU M., SLICHER A., KALLMAN K. D., 1965. Functionnal capacity of ectopic pituitary transplants in the teleost fish (Pœcilia formosa), with a comparative discussion on the transplanted pituitary. Phil. Trans. roy. Soc., London, 249B, 69-99.

BILLARD R., PETER R. E., 1977. Gonadotrophin release after implantation of anti-estrogens in the pituitary and hypothalamus of Goldfish, Carassius auratus. Gen. comp. Endocr., 32, 213-220.

BRETON B.,WEIL C., 1973. Effets du LH/FSH-RH synthétique et d'extraits hypothalamiques de Carpe sur la sécrétion d'hormones gonadotropes in vivo chez la carpe (Cyprinus carpio L.). C. R. Acad. Sci. Paris, série D, 277, 2061-2064.

CHAMBOLLE P., 1966. Recherches sur l'allongement de la durée de survie après hypophyseciomie chez Gambusia sp., C. R. Acad. Sci. Paris, 262, 1750-1753.

CHAMBOLLE P., 1977. Hypophyse et reproduction. Invesi. Pesq., 41, 1-13.

EKENGREN B., 1975. Structural aspects of the hypothalamo-hypophysial complex of the Roach, Leuciscus rutilus. Chem. Communic. Stokh., 29 p.

FRIDBERG G., EKENGREN B., 1977. The vascularization and the neuroendocrine pathways of the pituitary gland in the Atlantic Salmon, Salmo salar. Can. J. Zool., 55, 1284-1296.

GOOS H. J. TH., MURATHANOGLU O., 1977. Localisation of gonadotropin releasing hormone in the forebrain and neurohypophysis of the Trout Salmo gairdneri. An. immunofluorescence słudy. Cell Tiss. Res., 181, 163-168.

JOHANSEN P. H., 1967. The role of the pituitary in the resistance of the Goldfish (Carassius auratus L.) to high temperature. Con. J. Zool., 45, 329-345.

LU R. H., GRANDISON L., HUANG H. H., MARSHALL S., MEITES J., 1977. Relation of gonadotrophin secretion by pituitary grafts to spermatogenesis in hypophysectomized male rats. Endocrinology, 100, 380-386.

PETER R. E., 1970. Hypothalamic control of thyroid gland activity and gonadal activity in the Goldfish, Carassius auratus. Gen. comp. Endocr., 14, 334-356.

PEUTE J., DE BRUYN G. A., SELDENRIJK R., VAN OORDT P. G.W. J., 1976. Cyłophysiology and innervation of gonadotropic cells in the pituitary of the black-molly (Pœeilia latipinna). An electron microscopical study. Cell Tiss. Res., 174, 36-54.

SATHYANESAN A. G., 1970. Hypothalamo-hypophysial vascularization and its relationships with the neurosecretory system in the Catfish Clarias batrachus L. Zool. Beitr., 17, $1+11$.

SATHYANESAN A. G., 1971. Presence of hypothalamo-hypophyseal portal-system in the teleost Heferopneustes fossilis (B!.). Experientia, 27, 462.

SHERIDAN R., PASTEELS J. L., 1978. Autonomous secretion of FSH by rat pituitaries in long term organ culfure. Gen. comp. Endocr. 34, 74.

WEIL C., BRETON B., REINAUD P., 1975. Etude de la réponse hypophysaire a l'administration de Gn-RH exogène au cours du cycle reproducteur annuel chez la carpe Cyprinus carpio $L$. C. R. Acad. Sci. Paris, série D, 280, 2469-2472.

ZAMBRANO D., 1972. Innervation of the feleost pituitary. Gen. comp. Endocr., Suppl. 3, $22-31$. 